\title{
Degradation assessment of LYRA after 5 years on orbit - Technology Demonstration -
}

\author{
A. BenMoussa • B. Giordanengo • S. Gissot • \\ I. E. Dammasch • M. Dominique • J.-F. Hochedez • \\ A. Soltani • N. Bourzgui • T. Saito $・$ U. Schühle • \\ A. Gottwald • U. Kroth • A. R. Jones
}

Received: 12 July 2014 / Accepted: 22 December 2014 / Published online: 11 January 2015

C The Author(s) 2015. This article is published with open access at Springerlink.com

\begin{abstract}
We present a long-term assessment of the radiometric calibration and degradation of the Large Yield Radiometer (LYRA), which has been on orbit since 2009. LYRA is an ultraviolet (UV) solar radiometer and is the first space experiment using aboard a pioneering diamond detector technology. We show that LYRA has degraded after the commissioning phase but is still exploitable scientifically after almost 5 years on orbit thanks to its redundancy design and calibration strategy correcting for instrument degradation. We focus on the inflight detector's calibration and show that diamond photodetectors have not degraded while silicon reference photodiodes that are even less exposed to the Sun show an increase of their dark current and a decrease of their photoresponse.
\end{abstract}

A. BenMoussa $(\bowtie) \cdot$ B. Giordanengo $•$ S. Gissot $\cdot$ I. E. Dammasch $\cdot$ M. Dominique $\cdot$ J.-F. Hochedez Solar Terrestrial Center of Excellence, Circular 3, 1180 Brussels, Belgium

e-mail: ali.benmoussa@stce.be

J.-F. Hochedez

LATMOS-CNRS UMR 8190, UVSQ, UPMC, IPSL, 78280 Guyancourt, France

A. Soltani $\cdot$ N. Bourzgui

Institut d'Electronique, de Microélectronique et de Nanotechnologie (IEMN), 59652 Villeneuve d'Ascq, France

T. Saito

Department of Environment \& Energy, Tohoku Institute of Technology, Sendai, Miyagi 982-8577, Japan

U. Schühle

Max Planck Institute for Solar System Research, Justus-von-Liebig-Weg 3, 37077 Göttingen, Germany

A. Gottwald • U. Kroth

Physikalisch-Technische Bundesanstalt (PTB), Abbestr. 2-12, 10587 Berlin, Germany

A. R. Jones

Laboratory for Atmospheric and Space Physics, University of Colorado, 3665 Discovery Drive, Boulder, CO 80303, USA 
Keywords UV solar radiometer- Onboard calibration - Degradation - Diamond detector

\section{Introduction}

On November 2, 2009, the PROBA2 - Project for on Board Autonomy - satellite was launched into a Sun-synchronous polar orbit (altitude of $725 \mathrm{~km}$ ), which allows quasipermanent solar observations. PROBA2 is the second of the PROBA small satellites series, which is part of the European Space Agency (ESA)'s In orbit Technology Demonstration Program. PROBA2 incorporates a total of 17 new technological developments and four scientific experiments focused on solar and space weather observations. The LYRA instrument $[1,2]$ is the first ultraviolet (UV) solar radiometer in space that benefits from diamond photodetectors. It measures solar irradiances from soft Xray to UV spectral ranges, with a nominal cadence of $20 \mathrm{~Hz}$ (integration time of $50 \mathrm{~ms}$ ) that can be increased up to $100 \mathrm{~Hz}$. LYRA consists of three units operated as redundant radiometers so as to maximize the accuracy and the reliability of the measurements. Each unit contains four individual detection channels which consist of a collimator, a precision aperture, a large band spectral filter, a detector, and two light emitting diodes (LEDs) for in-flight detector (cf. Fig. 1).

The detectors are either silicon photodiodes (Si-AXUV, type AXUV20 from former International Radiation Detectors, now OptoDiode Corp.) or diamond detectors, the latter having been specifically designed for LYRA [3]. A detailed description of LYRA performances can be found in [4].

Since the time when its covers were opened in January 2010, LYRA has undergone a severe signal decrease that progresses with exposure to solar irradiation as shown in Fig. 2. Previous work has shown that the most likely cause of the observed degradation is contaminant buildup and UV-induced polymerization of organic molecules on the optical filters $[2,5]$. At UV (and shorter) wavelengths, molecular contamination on optical surfaces polymerizes and forms an absorbing layer. According to a recent contamination model [6] examining the 'as-built' assembly documents for the LYRA cover mechanism, both silicone and carbon (from epoxy and spacecraft outgassing [7]) constituents were identified as possible sources of contaminants.

In this paper, an assessment of the LYRA instrument's performance and degradation is presented based on our onboard calibration strategy which includes -1- detector dark current (DC) measurements with LYRA covers closed, -2- onboard Light Emitting Diodes (LEDs) signal acquisition to assess the detector stability and to disentangle aging of the detectors from the optical filters, -3-parallel data acquisition sequence (redundancy concept) where data from two LYRA units can be acquired in parallel to inter-calibrate their spectral channels and -4- inter-calibration planning which combines LYRA observations with currently operating UV solar instruments.

\section{Onboard detector calibration}

The radiometric calibration stability over years of mission time is a goal for a solar instrument such as LYRA. The bi-weekly onboard detector calibration sequence consists of 40 min of dark current (DC) measurement with the covers closed, followed 


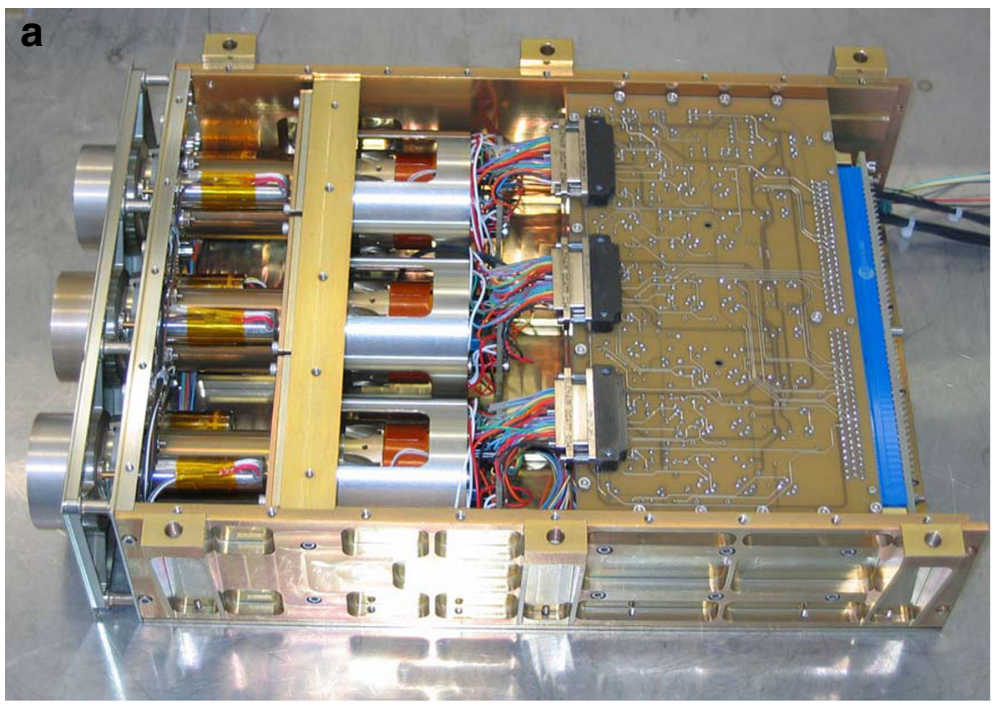

b

\section{1 unit $=4$ spectral channels}

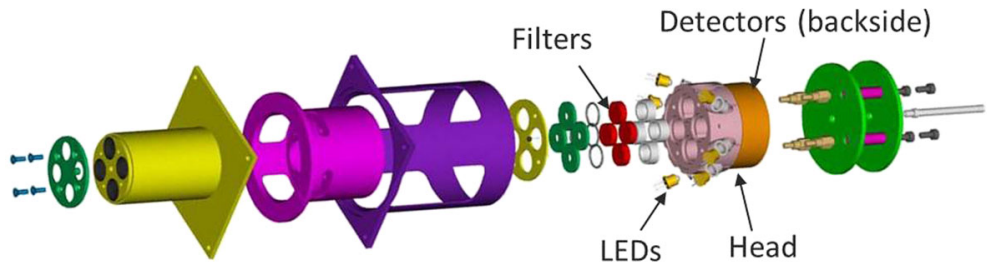

Fig. 1 a) LYRA instrument, and b) an exploded view of one of the 3 LYRA units including each four spectral channels

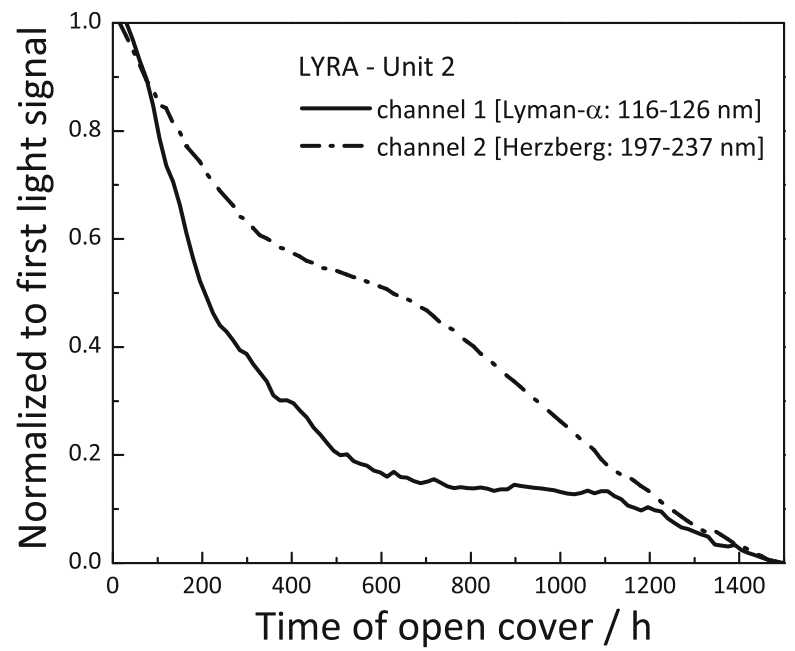

Fig. 2 Temporal degradation of LYRA unit 2 (channel 1 and 2) between 06 January and 30 March 2010 (first $1500 \mathrm{~h}$ ). Data are corrected from the dark current 
by 100 min with the visible (VIS) LED emitting at around $470 \mathrm{~nm}, 100 \mathrm{~min}$ with (UV) LED emitting at around $365 \mathrm{~nm}$ and again $40 \mathrm{~min}$ DC acquisition (see in Fig. 8). The sequence is first applied to the nominal unit 2 and to the first backup unit 3, after which this is repeated with unit 1. For LYRA, two types of diamond detectors had been developed by IMOMEC (University of Limburg, Belgium): metal-semiconductormetal (MSM) photoconductors [8] and PIN (with the n-doped layer on top) photodiodes [9]. Si-AXUV photodiodes were selected as reference detectors [10] for comparison with the newly developed diamond devices. All LYRA detectors had been measured regarding their quantum efficiency, stability, response uniformity and linearity showing good radiometric characteristics under laboratory conditions [4].

\subsection{Dark current measurements}

Assessing the dark current (DC) is an important calibration step. The DC is measured with LYRA covers closed before and after the science data acquisition with the same integration time. It is then subtracted from the integrated solar signal. After the first data acquisition in January 2010, the LYRA instrument temperature was varying between 27 and $33{ }^{\circ} \mathrm{C}$ all along the orbit due to the eclipse season that is imposed by the Sun synchronous orbit and which lasts every year from November to February. The operational temperature of LYRA has grown now (2014) up to $50{ }^{\circ} \mathrm{C}$, probably caused by the degradation of the spacecraft thermal surfaces, as under UV irradiation, contaminants will also form a polymerized film changing the spacecraft thermal control and affecting the payloads temperature [11]. As shown in Fig. 3, the DC of the diamond MSM increases exponentially with temperature, according to laboratory measurements. But it actually decreases significantly in space with the solar irradiation time (cf. inset in Fig. 3). As an example at $47^{\circ} \mathrm{C}$, ch $2-1$ (unit 2 - channel 1) shows a $32 \%$ decrease of its DC measured between S2 (2nd semester in 2010) and S9 (1st semester in 2014). It

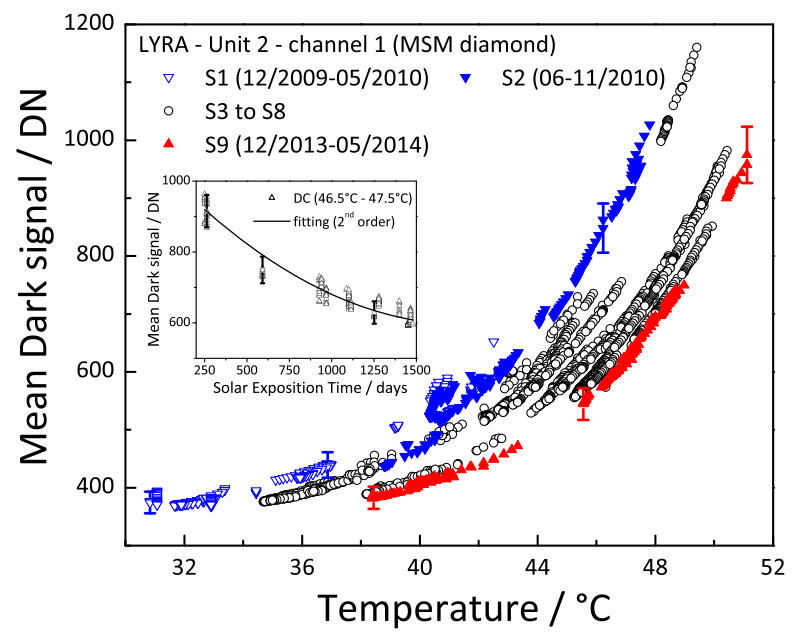

Fig. 3 Mean DC of diamond MSM detector (ch 2-1) as a function of the onboard temperature. The vertical scale (DN: digital number) is not calibrated. The inset shows the decrease of the DC as a function of the solar exposure time. Each dot is the average of 4000 data points acquired at $50 \mathrm{~ms}$ integration time. $\mathrm{S} 1$ is referring to the first semester period of LYRA on orbit, i.e., from December 2009 to May 2010 
should be mentioned that unit 2 is used continuously as the nominal unit. Unit 3 is much less exposed to the Sun than unit 2 while unit 1 remains closed most of the time (cf. Table 1).

The DC decrease in unit 2 is not fully understood. However, a possible explanation could be related to desorption of surface contaminants (mainly water) which increase conductivity. Due to the hydrogen-terminated MSM diamond surface [12] and the proximity of its interdigitated finger electrodes $(15 \mu \mathrm{m})$, diamond MSM detectors are very sensitive to the ambient environment and to surface contamination. Special precautions were taken to keep the diamond MSM detectors in a dry inert environment by purging LYRA continuously with nitrogen and keeping covers always closed during on-ground activities but this was perhaps not sufficient.

As shown in Table 1, diamond MSMs of unit 1, that are less exposed to the Sun, show a non-significant increase of their DC during the same period. The relative DC variation $(\triangle \mathrm{DC})$ is approximately $+4.0 \%$ at $47^{\circ} \mathrm{C}$ within the measurement uncertainty. It should be mentioned that the large error bar used, i.e., \pm 2 to $\pm 5 \%$, is mainly related to the time needed for each MSM detector to reach a stable signal. Indeed although diamond MSM detectors have negligibly small DC at room temperature (typically 1 to $2 \mathrm{pA}$ at $5 \mathrm{~V}$ bias voltage for a 5-mm diameter active area), they show a slow but persistent decrease of their DC, which varies with the cumulated duration and the flux of previous UV radiation exposures [8] but mainly with the electric field applied between the metal fingers electrodes. Solutions and progress have been already reported: new diamond MSM device architecture with optimized metal layers and with $5 \mu \mathrm{m}$ (instead of 15) spacing between the interdigitated finger contacts have been investigated and showed excellent results in terms of stability and homogeneity [13].

Diamond PIN photodiodes, in all three LYRA units, show high stability of their DC over a wide temperature range as shown in Fig. $4 . \triangle \mathrm{DC}$ is around $+0.2 \%$ at $47{ }^{\circ} \mathrm{C}$ which is regarded as insignificant. The fact that the PIN photodiodes operate in

Table 1 Relative variation of the dark current ( $\triangle D C$ ) measured between semester S1 (2010) and S9 (2014). FWHM: Full Width at Half Maximum of the filter transmission range. It should be mentioned that $\mathrm{Al}$ and $\mathrm{Zr}$ filters used in ch 3 and ch 4, respectively show high transmittance below 3.3 and $1.5 \mathrm{~nm}$ wavelength, respectively

\begin{tabular}{lllll} 
Unit - channel & Detector type & Bandwidth FWHM $/ \mathrm{nm}$ & $\Delta \mathrm{DC}\left(47^{\circ} \mathrm{C}\right) / \%$ & Cumul. solar exposure (days) \\
\hline $1-1$ & MSM diam. & $116-126$ & $+4.6 \pm 5$ & 7 \\
$1-2$ & PIN diam. & $197-237$ & $+0.2 \pm 0.2$ & 7 \\
$1-3$ & MSM diam. & $17-40(\mathrm{Al})$ & $+4.0 \pm 2$ & 7 \\
$1-4$ & Si-AXUV & $6-17(\mathrm{Zr})$ & $+2.0 \pm 2$ & 7 \\
$2-1$ & MSM diam. & $116-126$ & $-32 \pm 5$ & 1506 \\
$2-2$ & PIN diam. & $197-237$ & $+0.2 \pm 0.2$ & 1506 \\
$2-3$ & MSM diam. & $17-40$ & $-10 \pm 2$ & 1506 \\
$2-4$ & MSM diam. & $6-17$ & $-26 \pm 5$ & 1506 \\
$3-1$ & Si-AXUV & $116-126$ & $+14 \pm 2$ & 81 \\
$3-2$ & PIN diam. & $197-237$ & $+0.2 \pm 0.2$ & 81 \\
$3-3$ & Si-AXUV & $17-40$ & $+13.5 \pm 2$ & 81 \\
$3-4$ & Si-AXUV & $6-17$ & $+14 \pm 2$ & 81 \\
\hline
\end{tabular}




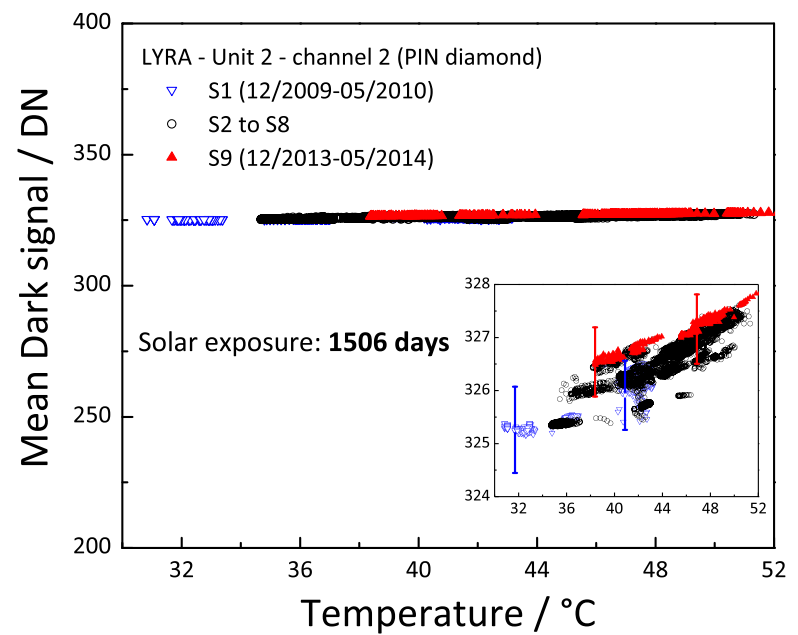

Fig. 4 Mean DC of diamond PIN photodiode (ch 2-2) as a function of the onboard temperature. The inset shows a close view

unbiased mode, i.e., do not require an external voltage, implies a very low DC of approximately $10^{-14} \mathrm{~A}$, as measured in the laboratory.

Si-AXUV photodiodes are used in channel 1, 3, and 4 in unit 3, and in channel 4 of unit 1 (cf. Table 1). They are based on n-on-p structure with a thin (6 to $-9 \mathrm{~nm}$ ) nitrided passivating silicon dioxide $\left(\mathrm{SiO}_{2}\right)$ layer for radiation hardness [14]. As shown in Fig. 5, Si-AXUV photodiodes from unit 3 show a much stronger increase of their DC $\left(\triangle \mathrm{DC}=+14 \%\right.$ at $\left.47^{\circ} \mathrm{C}\right)$ after 81 days of solar exposure as compared with unit 1 $\left(\triangle \mathrm{DC}=+2 \%\right.$ at $\left.47^{\circ} \mathrm{C}\right)$.

Different mechanisms could contribute to the DC increase of Si-AXUV photodiodes however it is well known that UV radiation as well as ionizing particles induce ionization damages in the $\mathrm{Si}-\mathrm{SiO}_{2}$ interface, resulting in the formation of oxidetrapped charges and interface states that affect the performance of the device $[15,16]$.

\subsection{LED measurements}

In order to test in-flight the detector stability, each LYRA channel carries along two LEDs. These LEDs emit radiation at $365 \mathrm{~nm}$ (UV - model NSHU551B from Nichia Corporation) and $470 \mathrm{~nm}$ (VIS - model 62612 from Micropac Industries) and are situated between the filter and detector as shown in Fig. 6, i.e., outside the optical path from the Sun viewed by the filter and the detector. The LEDs monitor the photoresponse of the Si-AXUV photodiodes in order to distinguish the detector's drift from the degradations of the filters transmission. Unfortunately, when LYRA was built, there existed no comparable compact light source to monitor the photoresponse of the diamond detectors (PIN and MSM) below their cut-off wavelength at around $225 \mathrm{~nm}$. However, the weak sub-bandgap photoresponse of diamond detectors can be usefully monitored with these LEDs despite the low efficiency at those wavelengths.

The MSM diamond detectors (except from ch 2-1) have been stable since the commissioning phase in December 2009, showing high stability with respect to the onboard LEDs. Indeed the (sub-bandgap) signal from ch 2-1 decreased slightly between 
Fig. 5 Mean DC as a function of the onboard temperature of two similar channels using Si-AXUV photodiodes a) ch 3-4 (unit 3) and b) ch 1-4 (unit 1)
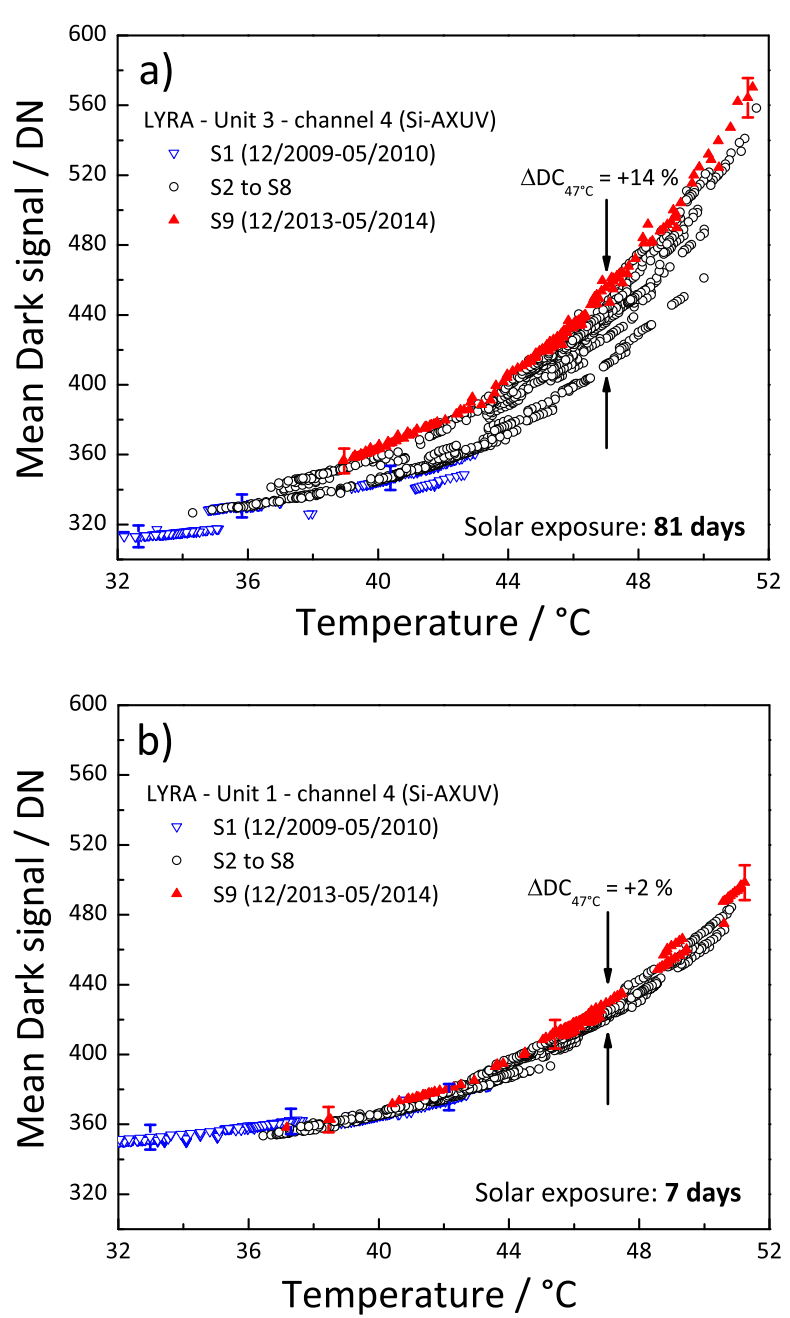

2010 and 2012 by approximately 12 and $8 \%$ for the VIS and UV LEDs respectively. Since 2012, the LED signal (after DC correction) is stable as shown in Fig. 7. Together with the previously mentioned decrease of its DC, the increase of its NUV-VIS rejection, confirms an enhancement of the ch 2-1 MSM diamond detector characteristics.

As shown in Fig. 8, diamond PIN photodiodes are slightly sensitive to the onboard LEDs although they exhibit a high rejection ratio of almost six orders of magnitude between 200 and $500 \mathrm{~nm}$ [9]. The stability of the (sub-bandgap) photoresponse when illuminated with the LEDs (e.g., $18 \pm 2$ DN with the VIS LED, cf inset of Fig. 8), together with a stable DC, demonstrates that the PIN diamond photodiodes have not degraded after almost 5 years on orbit.

Although all of the Si-AXUV photodiodes of unit 3 show the same DC increase (+ $14 \%$ at $47{ }^{\circ} \mathrm{C}$ ), the change of the photoresponse to the onboard LEDs differs from channel to channel. The responsivity change after UV solar exposure can depend on various parameters, e.g., the photon energy and flux. It is accepted that photons with 

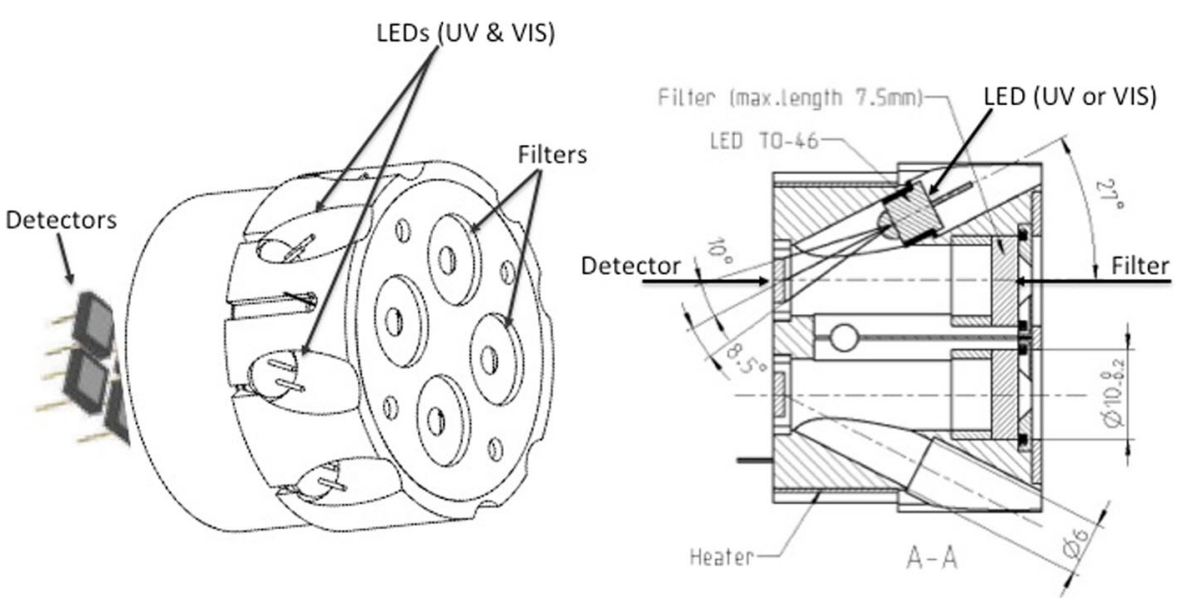

Fig. 6 Schematic drawing of a LYRA head (left) and its cross-section (right)

energies higher than the $9 \mathrm{eV}$ bandgap of $\mathrm{SiO}_{2}$ generates charges and consequently trap states at the $\mathrm{Si}-\mathrm{SiO}_{2}$ interface [17]. As shown in Fig. 9, the Si-AXUV photoresponse decreases with time at a given temperature. We observe a stronger photoresponse variation with the UV LED of -9 and $-7.5 \%$ at $47{ }^{\circ} \mathrm{C}$ for ch $3-3$ and $3-4$, respectively. This significant variation with the UV LED suggests that photogenerated carrier recombination occurs preferentially in the detector surface, either in the $\mathrm{Si}-\mathrm{SiO}_{2}$ interface or in the first atomic layers of the Si n-type region. Indeed UV LED photons are absorbed very close to the Si surface, i.e., $60 \%$ of them are absorbed in the first $10 \mathrm{~nm}$ [18] assuming that the oxide passivation layer is transparent. Similar wavelength dependency with a stronger decrease towards the NUV range during exposure to UV radiation has been reported in Si detector technology $[19,20]$. However as shown in Fig. 10, it is interesting to clarify why the Si-AXUV photodiode in ch 3-3 (but not in ch 3-4) shows a significant decrease of its photoresponse with its VIS LED by approximately $-5.5 \%$ at $47{ }^{\circ} \mathrm{C}$ (cf. Fig. 10a). For the VIS LED, the photons are absorbed

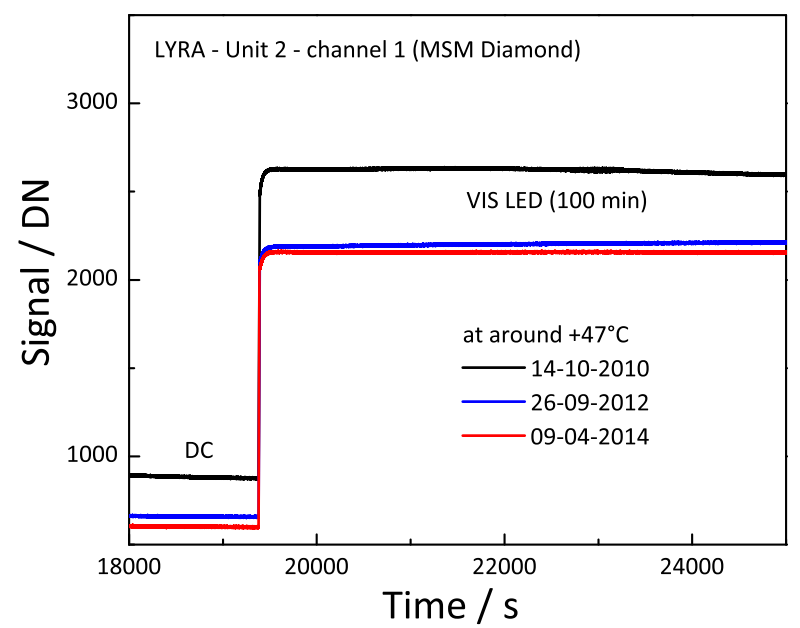

Fig. 7 Close view of the onboard calibration sequence of the MSM diamond detector (ch 2-1): DC followed by VIS LED (UV LED illumination is not shown) 


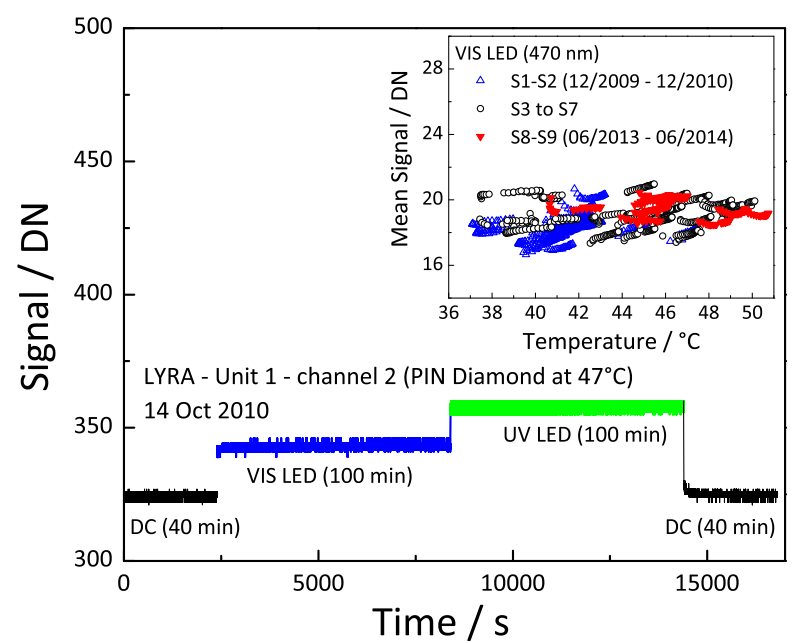

Fig. 8 Onboard calibration sequence of the PIN diamond photodiode (ch 1-2). The inset shows the subbandgap photoresponse as a function of temperature when illuminated by the VIS LED. The detector signal has been corrected for the dark current

deeper in the Si detector (the attenuation length is approximately $300 \mathrm{~nm}$ [18]) and consequently the collection of photogenerated charges should be less affected by the UV-induced surface damage as seen in Fig. $10 \mathrm{~b}$ with $-0.5 \%$ variation for ch $3-4$ at $47^{\circ} \mathrm{C}$. We suppose the higher signal variation in $\mathrm{ch} 3-3$ should be related to a depletion (bulk) damage caused by solar EUV photons with energies larger than the 21-eV threshold energy needed to create a displacement in Si material [21]. The difference in the degradation mechanism between those two EUV channels are likely related to the higher photon flux in the Al bandpass, i.e., 17 to $40 \mathrm{~nm}$ of ch 3-3, which includes the He II $30.4 \mathrm{~nm}$ solar emission line, the brightest line after hydrogen $(\mathrm{H})$ Lyman- $\alpha$. For ch 3-1, which monitors the Lyman- $\alpha$ bandpass between 116 and $126 \mathrm{~nm}$, SiAXUV photodiode has degraded only by 1.5 to $2.5 \%$ in the response to the UV and VIS LEDs (not shown). The stability of the Si-AXUV at the Lyman- $\alpha$ photon energy $(10.2 \mathrm{eV})$ has been reported elsewhere to exhibit only $3 \%$ drop in efficiency for a fluence of $10^{16}$ photons $/ \mathrm{cm}^{2}$ [22]. Si-AXUV photodiodes are thus more sensitive to high-energy EUV photon damage, resulting in collection efficiency loss. However, they show a better stability at the Lyman- $\alpha$ bandpass which is certainly related to its improved radiation-hard (nitrided) passivation layer [14].

It is important to mention that a stable sensitivity to the onboard LEDs does not exclude the presence of a UV radiation-induced thin surface contamination layer on the detector, which is transparent in the near UV and visible ranges but very absorptive in the EUV-VUV spectral range. Ideally, the responsivity change should be monitored over the spectral range in which the detector is used.

\section{Exploitation of the redundancy concept}

For the in-flight validation and eventual correction of the measured data, LYRA comprises three similar (although not -strictly identical) independent units. Two 
Fig. 9 Si-AXUV photoresponse as a function of temperature when illuminated by the UV LED for a) ch 3-3 and b) ch 3-4. The signal has been corrected for its dark current but not from the LED temperature dependence
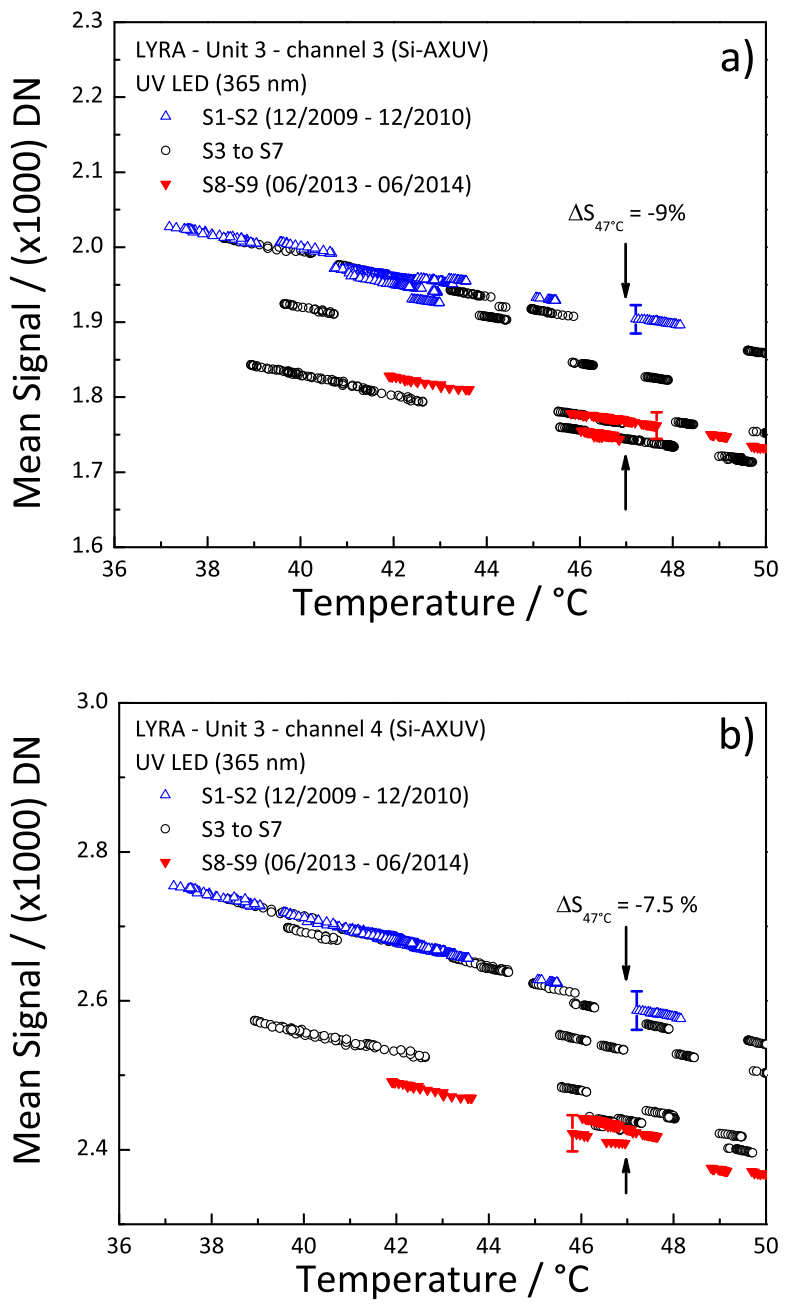

redundant groups of multiplexers and voltage-to-frequency converters (VFC) can be used to read one or two units in parallel to inter-calibrate the four spectral channels. The degraded channels from unit 2 can then be corrected by comparing the measured irradiances to those obtained by units 1 and 3 that have been less often exposed to the Sun. Since the beginning of January 2010, unit 2 has been observing the Sun continuously, while unit 3 is used on a daily basis during a short period, and unit 1 remains closed most of the time and is only used a few times during the mission (see in Table 1 for the cumulated solar exposure days). As an example, Fig. 11 shows the corrected temporal irradiance of channel 1 and 2 of unit 2 . The signal has been corrected from the dark current, and the degradation-trend has been removed and converted to physical units. Details of the correction methods are reported in [2].

It should be added that in order to reduce the uncertainty of the integrated signal, LYRA uses three reference calibration voltages $(0,2.5$ and $5 \mathrm{~V})$ to calibrate its VFCs (AD652). After almost 5 years in orbit, the 8 VFCs show good stability with time 

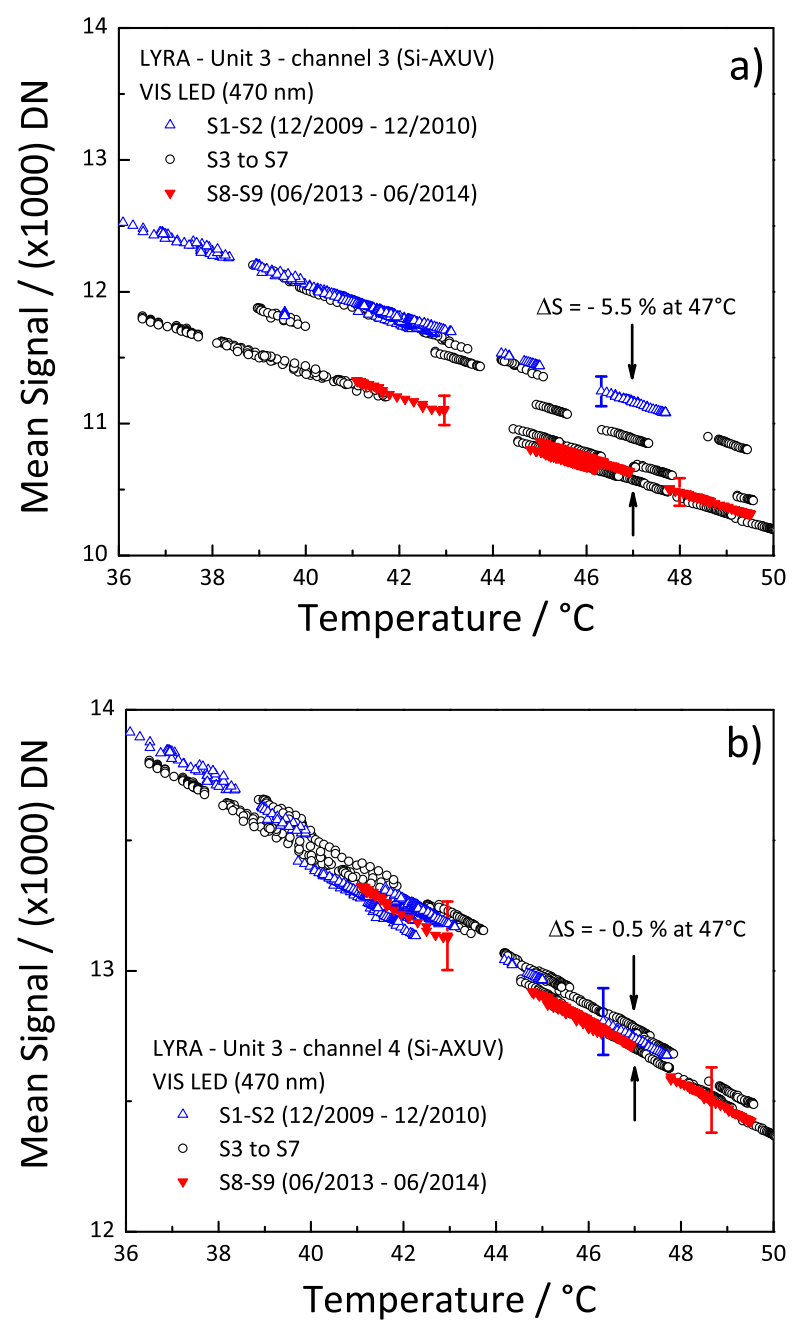

Fig. 10 Si-AXUV photoresponse as a function of temperature when illuminated by the VIS LED for a ch 3-3 and $\mathbf{b}$ ch 3-4. The signal has been corrected for its dark current but not from the LED temperature dependence

(relative variation of $0.029 \%$ at $5 \mathrm{~V}$ ) and also as a function of the temperature $(1.45$ $10^{-3} \% / \mathrm{K}$ at $\left.5 \mathrm{~V}\right)$.

\section{Inter-calibration}

Long-term UV solar irradiance measurement can only be achieved by combining observations of several space-based instruments. Since January 2010 LYRA has been continuously observing the Sun and, after correction, it could be calibrated with the help of a combined solar spectrum by the Solar Stellar Irradiance Comparison Experiment (SOLSTICE) onboard the Solar Radiation and Climate Experience (SORCE) and the Solar EUV Experiment (SEE) on the Thermosphere Ionosphere Mesosphere Energetics and Dynamics (TIMED) mission. In addition, first results indicated that 


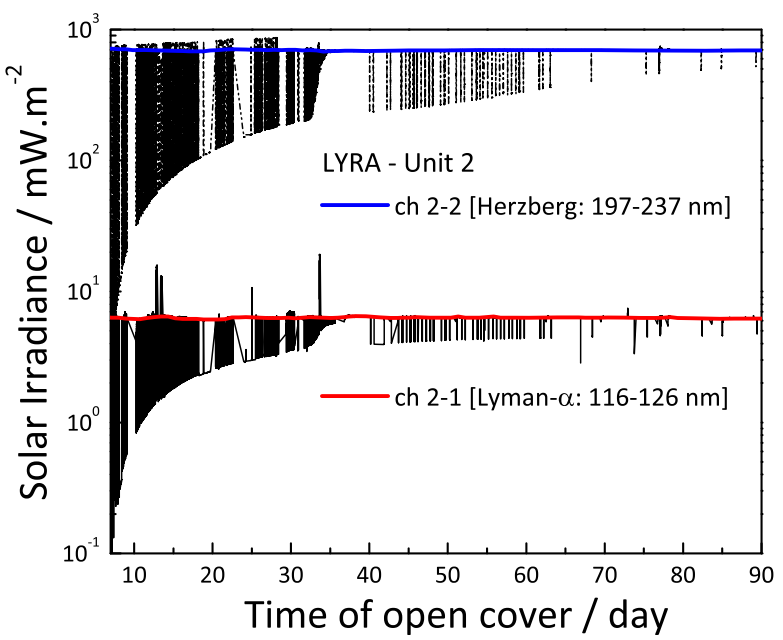

Fig. 11 Corrected solar irradiance $\left(\mathrm{mW} / \mathrm{m}^{2}\right)$ of LYRA ch 1-2 and 2-2 (unit 2). The color lines are a guide to the eye. The black curved background corresponds to the additive degradation correction. The transitions between full Sun / no Sun are due to the eclipse season

the spatial resolution of SWAP on PROBA2 complements well the high temporal resolution of the EUV LYRA channels [23]. SWAP — the Sun Watcher using APS and image Processing - is an EUV solar telescope centered at $17.4 \mathrm{~nm}$ and the first on orbit that benefits from CMOS Active Pixel Sensor technology [24, 25]. The long-term variations of the EUV irradiance measured by LYRA are also compared with the EUV Variability Experiment (EVE) on board the Solar Dynamics Observatory (SDO) satellite [26]. It has been shown that the LYRA channel 2-4 data correlate well with the EVE/ESP (EUV Spectro-Photometer) level-1 data between 2010 and 2013 [27]. LYRA channel 2-4 is also in good agreement with the EVE/MEGS (Multiple EUV Grating Spectrograph) channels [28]. However, LYRA channel 2-3 has shown a much smaller variability due to the degradation of the longer wavelengths included in its passband i.e., above approximately $19 \mathrm{~nm}$. A new inter calibration campaign with EVE/MEGS is under study with improved degradation correction of LYRA channels and will be the subject of a future publication.

The LYRA proxy data for the EUV channels and the soft X-ray solar flux of NOAA's Geostationary Operational Environmental Satellites (GOES) are shown in Fig. 12.

The calibrated data (in physical units), corrected from the temperature effects DC, and degradation-trends are available on the LYRA website (proba2.sidc.be/data/ LYRA).

\section{Conclusions}

Since 2010, LYRA has been exposed to a severe environment and it has consequently experienced significant degradation. The observed degradation is caused mainly by the presence of contaminant species (organics, siloxanes and water) on the optical filter 


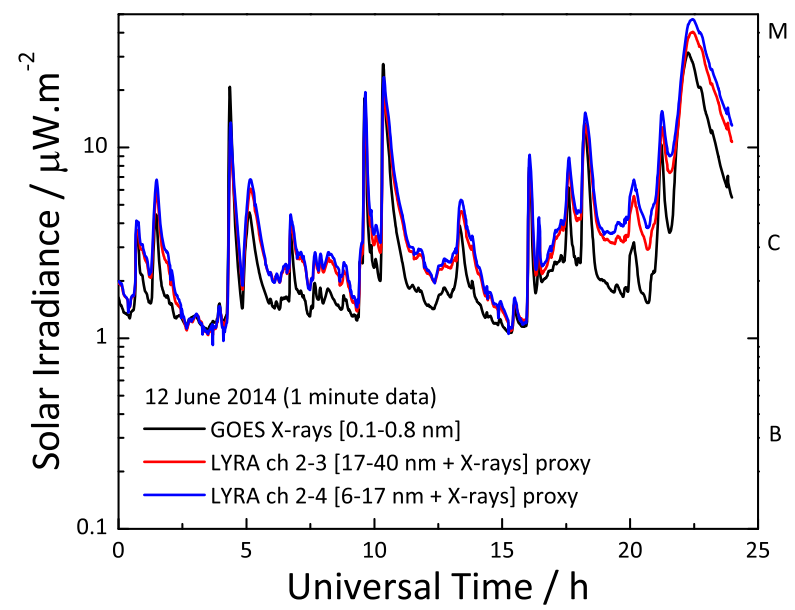

Fig. 12 Comparison of the LYRA ch 2-3 and 2-4 proxies with GOES X rays solar flux. Note that Al (ch 2-3) and $\mathrm{Zr}$ (ch 2-4) filters show high transmittance below 3.3 and $1.5 \mathrm{~nm}$ wavelength, respectively

surface, which reduce the UV light transmission. Currently, there is no possibility of mitigating contamination in space once molecules have settled irreversibly after UV-polymerization, which is a classical concern for UV solar instruments. However, methods to largely recover the LYRA calibration were established and successfully implemented. In-flight detector monitoring shows that diamond detectors (both MSM and PIN) exhibit insignificant degradation after almost 5 years on orbit. By its nature, diamond has a much lower thermally induced DC and is a radiation-hard semiconductor, which significantly extend the stability of the LYRA radiometric calibration. Conversely, and despite a much lower duty cycle, the SiAXUV photodiodes used as reference detectors show an increase of their dark current and a higher decrease of their photoresponse, as demonstrated by the onboard UV LEDs for the EUV LYRA channels, suggesting damage in the oxide passivation layer. Exposure to radiation is often the main reason for detector degradation, and its impacts (ionization and displacement-damage) are frequently underestimated. LYRA profits additionally from a redundancy design concept for tracking instrument degradation. Complemented by other solar inter-calibration instruments, LYRA started its new real-time space weather services. Finally, the successful PROBA2 technological mission offers a unique opportunity to validate diamond detector technology from which lessons are derived for future solar UV radiometers.

Acknowledgments LYRA is a project of the Centre Spatial de Liège, the Physikalisch-Meteorologisches Observatorium Davos and the Royal Observatory of Belgium funded by the Belgian Federal Science Policy Office (BELSPO) and by the Swiss Bundesamt für Bildung und Wissenschaft. This work was made possible thanks to the Solar-Terrestrial Centre of Excellence (STCE), a collaborative framework funded by BELSPO.

Open Access This article is distributed under the terms of the Creative Commons Attribution License which permits any use, distribution, and reproduction in any medium, provided the original author(s) and the source are credited. 


\section{References}

1. Hochedez, J.-F., Schmutz, W., Stockman, Y., Schühle, U., BenMoussa, A., Koller, S., Haenen, K., Berghmans, D., Defise, J.-M., Halain, J.-P., Theissen, A., Delouille, V., Slemzin, V., Gillotay, D., Fussen, D., Dominique, M., Vanhellemont, F., McMullin, D., Kretzschmar, M., Mitrofanov, A., Nicula, B., Wauters, L., Roth, H., Rozanov, E., Ruedi, I., Wehrli, C., Soltani, A., Amano, H., Van der Linden, R., Zhukov, A., Clette, F., Koizumi, S., Mortet, V., Remes, Z., Petersen, R., Nesladek, M., D’Olieslaeger, M., Roggen, J., Rochus, P.: LYRA, a solar UV radiometer on PROBA 2. Adv. Space Res. 37, 303 (2006)

2. Dominique, M., Hochedez, J.-F., Schmutz, W., Dammasch, I.E., Shapiro, A.I., Kretzschmar, M., Zhukov, A., Gillotay, D., Stockman, Y., BenMoussa, A.: The LYRA instrument on-board PROBA2: description and in-fight performances. Sol. Phys. 286(1), 21-42 (2013)

3. BenMoussa, A., Hochedez, J.F., Schühle, U., Schmutz, W., Haenen, K., Stockman, Y., Soltani, A., Scholze, F., Kroth, U., Mortet, V., Theissen, A., Laubis, C., Richter, M., Koller, S., Defise, J.-M., Koizumi, S.: Diamond detectors for LYRA, the solar VUV radiometer on board PROBA2. Diam. Relat. Mater. 15, 802-806 (2006)

4. BenMoussa, A., Dammasch, I.E., Hochedez, J.F., Schühle, U., Koller, S., Gillotay, Stockman, Y., Scholze, F., Richter, M., Kroth, U., Laubis, C., Dominique, M., Kretzschmar, M., Mekaoui, S., Gissot, S., Theissen, A., Giordanengo, B., Bolsee, D., Hermans, C., Gillotay, D., Defise, J.-M., Schmutz, W.: Pre-flight calibration of LYRA, the solar VUV radiometer on board PROBA2. Astron. Astrophys. 508, 10851094 (2009)

5. BenMoussa, A., Gissot, S., Schühle, U., et al.: On orbit degradation of solar instruments. Sol. Phys. 288(1), 389-434 (2013)

6. Jones, A.R., McMullin, D., Dominique, M., Dammasch, I.E.: Progress towards understanding the degradation and performance characteristics of the PROBA2-LYRA instrument, American Geophysical Union (AGU), Fall Meeting 2013, San Francisco, United States, 9-12 December 2013

7. Schläppi, B., et al.: Influence of spacecraft outgassing on the exploration of tenuous atmospheres with in situ mass spectrometry. J. Geophys. Res. 115, A12313 (2010)

8. BenMoussa, A., Theissen, A., Scholze, F., Hochedez, J.F., Schühle, U., Schmutz, W., Haenen, K., Stockman, Y., Soltani, A., McMullin, D., Vest, R.E., Kroth, U., Laubis, C., Richter, M., Mortet, V., Gissot, S., Delouille, V., Dominique, M., Koller, S., Halain, J.P., Remes, Z., Petersen, R., D’Olieslaeger, M., Defise, J.-M.: Performance of diamond detectors for VUV applications. Nucl. Inst. Methods A 568, 398-405 (2006)

9. BenMoussa, A., Schühle, U., Scholze, F., Kroth, U., Haenen, K., Saito, T., Campos, J., Koizumi, S., Laubis, C., Richter, M., Theissen, A., Hochedez, J.F.: Radiometric characteristics of new diamond pinphotodiodes. Meas. Sci. Technol. 17, 913-917 (2006)

10. Canfield, L.R., Vest, R.E., Korde, R., Schmidtke, H., Desor, R.: Absolute silicon photodiodes for $160 \mathrm{~nm}$ to $254 \mathrm{~nm}$ photons. Metrologia 35, 329-334 (1998)

11. Miller, S.K.R., Banks, B.: Degradation of spacecraft materials in the space environment. MRS Bull. 35, 20-24 (2010)

12. Kawarada, H.: Hydrogen-terminated diamond surfaces and interfaces. Surf. Sci. Rep. 26, 205-259 (1996)

13. BenMoussa, A., Soltani, A., Haenen, K., Kroth, U., Mortet, V., Barkad, H.A., Bolsee, D., Hermans, C., Richter, M., De Jaeger, J.C., Hochedez, J.F.: New developments on diamond photodetector for VUV solar observations. Semicond. Sci. Technol. 23, 035026 (2008)

14. Korde, R., Cable, J., Canfield, R.: One gigarad passivating nitrided oxides for $100 \%$ internal quantum efficiency silicon photodiodes. IEEE Trans. Nucl. Sci. 40, 1655-1659 (1993)

15. Homes-Siedle, A., Adams, L.: Handbook of Radiation Effects, 2nd edn. U.K. Univ. Press, Oxford (2002)

16. Shi, L.: Performance analysis of si-based ultra-shallow junction photodiodes for UV Radiation Detection, $\mathrm{PhD}$ Thesis, Delft University of Technology (NL) (2013)

17. Ma, T.P., Dressendorfer, P.V.: Ionization radiation effects in MOS devices and circuits. Wiley, New York (1989)

18. Palik, E.D. (ed.): Handbook of Optical Constants of Solids. Academic, New York (1985)

19. Shaw, P.-S., Gupta, R., Lykke, K.R.: Stability of photodiodes under irradiation with a 157-nm pulsed excimer laser. Appl. Opt. 44(2), 197-207 (2005)

20. Werner, L.: Ultraviolet stability of silicon photodiodes. Metrologia 35, 407-411 (1998)

21. Leroy, C., Rancoita, P.G.: Particle interaction and displacement damage in silicon devices operated in radiation environments. Rep. Prog. Phys. 70, 493-625 (2007) 
22. Gullikson, E.M., Korde, R., Canfield, L.R., Vest, R.E.: Stable silicon photodiodes for absolute intensity measurements in the VUV and soft x-ray regions. J. Electron Spectrosc. Relat. Phenom. 80, 313-316 (1996)

23. Kumara, S.T., Kariyappa, R., Dominique, M., Berghmans, D., Dame, L., Hochedez, J.F., Doddamani, V.H., Chitta, L.P.: Preliminary results on irradiance measurements from Lyra and swap. Adv. Astron. 2012, 1-5 (2012)

24. Seaton, D.B., Berghmans, D., Nicula, B., Halain, J.-P., De Groof, A., Thibert, T., Bloomfield, D.S., Raftery, C.L., Gallagher, P.T., Auchère, F., Defise, J.-M., D’Huys, E., Lecat, J.-H., Mazy, E., Rochus, P., Rossi, L., Schühle, U., Slemzin, V., Yalim, M.S., Zender, J.: The SWAP EUV imaging telescope part I: instrument overview and pre-flight testing. Sol. Phys. 286(1), 43-65 (2013)

25. Halain, J.P., Berghmans, D., Seaton, D.B., Nicula, B., De Groof, A., Mierla, M., Mazzoli, A., Defise, J.M., Rochus, P.: The SWAP EUV imaging telescope. Part II: in-flight performance and calibration. Sol. Phys. 286(1), 67-91 (2013)

26. Woods, T.N., Eparvier, F.G., Hock, R., Jones, A.R., Woodraska, D., et al.: Extreme ultraviolet variability experiment (EVE) on the solar dynamics observatory (SDO): overview of science objectives, instrument design, data products, and model developments. Sol. Phys. 275, 115-143 (2012)

27. Yalim, M.S., Poedts, S.: Variations in EUV irradiance: comparison between LYRA, ESP and SWAP integrated flux. Adv Astron. 2014, Article ID 957461, 13 pages (2014)

28. Kretzschmar, M., Dammasch, I.E., Dominique, M., Zender, J., Cessateur, G., D’Huys, E.: Extreme ultraviolet solar irradiance during the rising phase of solar cycle 24 observed by PROBA2/LYRA. J. Space Weather Space Clim. 2, A14 (2012) 